\title{
Depressive Symptoms in Relation to Clinical Symptom Onset of Mild Cognitive Impairment
}

\author{
Carol K. Chan ${ }^{1}$, Anja Soldan ${ }^{2}$, Corinne Pettigrew ${ }^{2}$, Mei-Cheng Wang ${ }^{3}$, Jiangxia Wang ${ }^{3}$, \\ Marilyn S. Albert ${ }^{2}$, Paul B. Rosenberg ${ }^{1}$, and the BIOCARD Research Team \\ ${ }^{1}$ Department of Psychiatry, Johns Hopkins University School of Medicine, Baltimore, MD, USA \\ ${ }^{2}$ Department of Neurology, Johns Hopkins University School of Medicine, Baltimore, MD, USA \\ ${ }^{3}$ Department of Biostatistics, Johns Hopkins University School of Public Health, Baltimore, MD, \\ USA
}

\section{Abstract}

Objective-There is increasing evidence of an association between depressive symptoms and mild cognitive impairment (MCI) in cross-sectional studies, but the longitudinal association between depressive symptoms and risk of MCI onset is less clear. The authors investigated whether baseline symptom severity of depression was predictive of time to onset of symptoms of MCI.

Method-These analyses included 300 participants from the BIOCARD study, a cohort of individuals who were cognitively normal at baseline (mean age $=57.4$ years) and followed for up to 20 years (mean follow-up $=12.5$ years). Depression symptom severity was measured using the Hamilton Depression Scale (HAM-D). The authors assessed the association between dichotomous and continuous HAM-D and time to onset of MCI within 7 years vs after 7 years from baseline (reflecting the mean time from baseline to onset of clinical symptoms in the cohort) using Cox regression models adjusted for gender, age, and education.

Results-At baseline, subjects had a mean HAM-D score of 2.2 ( $\mathrm{SD}=2.8$ ). Higher baseline HAM-D scores were associated with an increased risk of progression from normal cognition to clinical symptom onset $\leq 7$ years from baseline $(p=0.043)$, but not with progression $>7$ years from baseline $(\mathrm{p}=0.194)$. These findings remained significant after adjustment for baseline cognition.

Conclusions-These results suggest that low levels of depressive symptoms may be predictive of clinical symptom onset within approximately 7 years among cognitively normal individuals, and may be useful in identifying persons at-risk for MCI due to Alzheimer's disease.

Address Correspondence to Carol Chan, cchan46@jhmi.edu, 600 N Wolfe Street, Baltimore, MD 21287, Phone: 443-343-2060, Fax: 410-614-5914.

Disclosures: Dr. Marilyn Albert is an advisor to Eli Lilly. The following authors report no competing interests: Dr. Carol Chan, Dr. Anja Soldan, Dr. Corinne Pettigrew, Dr. Mei-Cheng Wang, Ms. Jiangxia Wang, Dr. Paul Rosenberg.

Description Of Author's Roles: C. Chan searched the literature, interpreted the results and drafted the paper. A. Soldan and C. Pettigrew contributed to statistical design, critical revisions, and interpretation of results. M. Wang and J. Wang performed the statistical analysis. M. Albert supervised the data collection, contributed to study design, interpretation of the results and critical revisions. P. Rosenberg supervised, contributed to statistical design, interpretation of the results, and critical revisions. All authors approved the final version of the paper for submission. 


\section{Keywords}

Mild cognitive impairment; Alzheimer's disease; depression; cognitive impairment

\section{INTRODUCTION}

Alzheimer's disease (AD) is the most common neurodegenerative disease, affecting an estimated 5 million persons in the U.S. alone, a number estimated to triple by the year 2050 (Alzheimer's Association 2017). Accumulating evidence indicates that the underlying neuropathological mechanisms associated with $\mathrm{AD}$ begin a decade or more before the emergence of clinical symptoms (Sperling et al., 2011). Prior studies have indicated that several types of measures, acquired when individuals are cognitively normal, are associated with increased risk of progressing to the early symptomatic phase of $\mathrm{AD}$, commonly referred to as mild cognitive impairment (MCI) (Petersen et al., 1999). These measures include cognitive testing, cerebrospinal fluid (CSF), and magnetic resonance imaging (MRI) (Albert et al., 2018). Less is known about the relationship of neuropsychiatric symptoms to risk of MCI.

Several neuropsychiatric symptoms, in particular apathy, anxiety and depression, have been identified as risk factors for progression from MCI to AD dementia (Palmer et al., 2010; Teng et al., 2007; Gabryelewicz et al., 2007). Even minimal severity of depressive symptoms has been associated with increased risk of progression from MCI to dementia (Rosenberg et al., 2013). A number of cross-sectional studies have found an increased prevalence of neuropsychiatric symptoms in patients with MCI compared to healthy controls (Lyketsos et al., 2002; Geda et al., 2008; Hwang et al., 2004). Depressive symptoms, specifically, have been correlated with functional impairment among individuals with MCI (Feldman et al., 2004).

Prospective studies of non-demented individuals have also found an association between depression and development of AD-dementia (Saczynski et al., 2010; Wilson et al., 2008). In particular, late-life depression as opposed to earlier depression has been reported to be associated with increased risk of progression to dementia (Li et al., 2011).

Longitudinal studies examining the relationship between baseline depression and risk of progression from normal cognition to MCI, however, have yielded mixed results. Some studies have reported an association between depressive symptoms in cognitively normal individuals and greater incidence of MCI (Barnes et al., 2006; Ravaglia et al., 2008) while others have not (Panza et al., 2008; Dotson et al. 2010). Two studies have reported a relationship between depressive symptoms among individuals with normal cognition at baseline and time to onset of MCI (Steenland et al., 2012; Wilson et al., 2008). However, participants in these studies were followed for a limited time period (ranging from $2.5-5$ years), making it difficult to examine the timing of these relationships.

The goal of this study was to determine whether baseline symptoms of depression were predictive of time to onset of symptoms of $\mathrm{MCI}$ in a cognitively normal, primarily middleaged cohort. The long follow-up of the cohort (mean $=12.5$ years, $\max =20$ years), relative 
to previous studies, allowed us to examine the impact of duration of follow-up on these relationships. Since the participants in the study had detailed cognitive assessments, we were also able to determine whether any associations observed were independent of baseline cognitive performance. The latter is particularly important since cognitive impairment is a known feature of late-life depression (Burt et al., 1995) which can persist even after the remission of mood symptoms (Bhalla et al., 2006).

\section{METHODS}

\section{Study Design and Participant Selection}

Data for this study were derived from the Biomarkers for Older Controls at Risk for Alzheimer's Disease (BIOCARD) study, which was designed to recruit and follow a cohort of cognitively normal individuals to identify variables that could predict the subsequent development of mild to moderate symptoms of AD. By design, approximately $75 \%$ of the participants had a first degree relative with dementia secondary to AD.

The BIOCARD study was initiated at the National Institutes of Health (NIH) in 1995. Recruitment procedures, baseline evaluations and annual clinical and cognitive assessments have been previously presented in detail (Albert et al., 2014). Briefly, recruitment was conducted by staff at the National Institutes of Mental Health Geriatric Psychiatry Branch. At baseline, participants completed a comprehensive evaluation consisting of a physical, neurological and psychiatric examination, neuropsychological testing, an electrocardiogram, and standard laboratory studies. Individuals were excluded from participation if they were cognitively impaired or had significant medical problems.

After providing written informed consent, a total of 349 individuals were enrolled in the study. While the initial study was at the NIH, participants were administered a comprehensive neuropsychological battery annually. Magnetic resonance imaging (MRI) scans, cerebrospinal fluid (CSF) samples, and blood specimens were obtained approximately every 2 years.

The study was stopped in 2005 for administrative reasons. In 2009, a research team from The Johns Hopkins School of Medicine was funded to re-establish the cohort, continue annual clinical and cognitive assessments, and evaluate the previously acquired data. Additional data collection was more recently initiated, including the collection of both MRI and CSF biomarkers and amyloid position emission tomography (PET) imaging in 2015, and tau PET imaging in 2017. See Figure 1 published as supplementary material online attached to the electronic version of this paper at https://www.cambridge.org/core/journals/ international-psychogeriatrics for a schematic representation of the study design. The analyses presented here are based on data from 300 subjects who were cognitively normal at baseline (see supplementary section 1 for the reasons subjects were excluded from the analyses).

\section{Clinical Assessments}

Clinical and cognitive assessments were completed annually at the NIH initially, and subsequently at Johns Hopkins, as noted above. A consensus diagnosis for each study visit 
was established by the staff of the BIOCARD Clinical Core at Johns Hopkins (prospectively for subjects evaluated starting in 2009 and retrospectively for subjects evaluated at the NIH). As previously described (Albert et al., 2014), each consensus diagnosis was handled in a similar manner. First a syndromic diagnosis was established: 1) clinical data pertaining to the medical, neurologic and psychiatric status of the subject were examined, 2) reports of changes in cognition by the subject and by a collateral source were reviewed, and 3) decline in cognitive performance, based on review of longitudinal testing from multiple domains (and by comparison with published norms) was determined. If a subject was deemed to be impaired, the decision about the likely etiology of the syndrome was based on the medical, neurologic, and psychiatric information collected at each visit, as well as medical records obtained from the subject, where necessary. More than one etiology could be endorsed for each subject (e.g., Alzheimer's disease and vascular disease). The consensus diagnosis procedures followed the diagnostic recommendations incorporated in the National Institute on Aging\& Alzheimer's Association (NIA-AA) workgroup reports for the diagnosis of MCI (Albert et al., 2011) and dementia due to AD (McKhann et al., 2011).

The estimated age of onset of clinical symptoms was established separately, based primarily on a semi-structured interview with the subject and the collateral source, derived from the Clinical Dementia Rating scale (Morris, 1993). See supplementary section 2 for additional details regarding diagnostic procedures.

\section{Cognitive Assessments}

The annual, comprehensive neuropsychological battery covered all major cognitive domains, including memory, executive function, language, visuospatial ability, attention, speed of processing and psychomotor speed (see Albert et al., 2014 for the complete battery). In the present analyses, we selected 4 measures that were previously identified to be the best combination of cognitive predictors of the time to progression from normal cognition to clinical symptom onset (Albert et al., 2014). These measures included 1) Paired Associates immediate recall of the Wechsler Memory Scale-Revised, 2) Logical Memory delayed recall (Story A) of the Wechsler Memory Scale-Revised, 3) Boston Naming, and 4) Digit-Symbol Substitution from the Wechsler Adult Intelligence Scale-Revised. A baseline cognitive composite score was calculated by transforming these 4 measures to $z$ scores and then averaging them, with the requirement that at least 2 of the 4 scores were present at a given time point ( $\mathrm{n}=279$ subjects with cognitive composite scores).

\section{Depression Assessment}

Assessment of depression symptomatology was measured at baseline with the Hamilton Depression Scale (HAM-D) (Hamilton, 1960). The HAM-D is a clinician\& administered scale consisting of 21 items. The instrument measures somatic and affective symptoms of depression. Each item is scored for severity on a scale of 0-2 or 0-5 points, with a higher score reflecting higher symptom severity. Scores of 0-7 are generally considered to be normal (Frank et al., 1991). The staff conducting the consensus diagnoses were blinded to the HAM-D scores of the participants. In these analyses, HAM-D scores were treated both as a continuous measure and were also dichotomized at HAM-D >1 vs. HAM-D 0-1. 1 was 
selected as the cutoff for dichotomous classification as it reflected the median HAM-D score in the cohort.

\section{Statistical Analysis}

We first compared group differences (i.e., remained normal vs. progressed to MCI) in baseline characteristics using $\mathrm{t}$-tests for continuous variables and chi-square tests for dichotomous variables.

Cox regression models (i.e., proportional hazard models) were then used to determine whether baseline HAM-D scores were associated with time (in years) to clinical symptom onset. To examine whether depression score severity was associated with clinical symptom onset for transitions occurring closer in time to baseline compared to those occurring more distally in time, we ran additional Cox regression models that included an indicator variable for time from baseline (within 7 years) as part of the coefficient for the depression measure. 7 years was selected as the cut-off point as it reflected the mean time to clinical symptom onset of MCI in the cohort (see Results), and had been used in previous similar analyses (Pettigrew et al., 2016). This analytic approach is equivalent to treating depression as a composition of two time-dependent variables, one having an effect on risk of progression within 7 years, and the other after 7 years (Fisher and Lin, 1999); this allowed us to examine whether baseline depression scores were differentially associated with risk of progression within 7 years vs. after 7 years from baseline. All Cox models were adjusted for baseline age, education, and gender. In a subset of models, baseline cognitive composite scores were included as an additional covariate to ensure that any findings related to HAM-D scores were not confounded with differences in cognition. The Schoenfeld residual method was used to check the proportional hazard assumption for the Cox regression models (see Supplementary section 3).

We also tested whether the association between baseline HAM-D scores and risk of clinical onset differed by gender by including gender x HAM-D interaction terms. Thus, if a significant gender $\mathrm{x}$ HAM-D interaction were found, it would be possible to conduct separate Cox regression models for males and females.

\section{RESULTS}

Table 1 shows baseline characteristics for the entire BIOCARD cohort ( $\mathrm{N}=349)$, and the subjects in the present analyses $(n=300)$, divided into participants who progressed to MCI $(n=69)$ vs. those who remained normal over time $(n=231)$. A subset of participants diagnosed as 'Impaired not MCI' $(\mathrm{n}=39)$ were included in the group with normal cognition (see supplementary section 2 for further details).

At baseline, participants who progressed to MCI were significantly older than those who remained cognitively normal $(\mathrm{p}<.001)$. The mean HAM-D score at baseline was $2.2(\mathrm{SD}=$ 2.8), far below 8 , the commonly used threshold for diagnosis of major depression (Kriston and von Wolff, 2011). Baseline HAM-D scores did not differ between groups. However, baseline cognitive composite scores were significantly lower in subjects who later progressed to MCI. 
In the Cox regression models, HAM-D scores at baseline were not associated with time to onset of clinical symptoms of MCI when the length of time between baseline and clinical symptom onset was not considered (continuous HAM-D: $\mathrm{HR}=0.88,95 \% \mathrm{CI}=(0.72,1.08)$, $\mathrm{p}=0.224$; dichotomous HAM-D: $\mathrm{HR}=0.74,95 \% \mathrm{CI}=(0.32,1.61), \mathrm{p}=0.422)$.

The mean time from baseline to clinical symptom onset for the participants who progressed to $\mathrm{MCI}$ was 7.5 years $(\mathrm{SD}=3.9)$, as noted above. Table 2 shows baseline characteristics of the cognitively normal participants who progressed to MCI, stratified by clinical symptom onset within 7 years from baseline vs. after 7 years from baseline. Baseline HAM-D scores were higher in participants who progressed proximally to baseline (i.e., within 7 years), vs. those who progressed more distally from baseline $(\mathrm{p}=.035)$. Importantly, the Cox regression model that included an indicator variable for time from baseline (within 7 years) showed that baseline continuous HAM-D scores were significantly associated with time to onset of clinical symptom for progression within 7 years of baseline $(p=.043)$, but not after 7 years (Table 3). Baseline dichotomous HAM-D scores were not associated with time to onset of clinical symptom of MCI. The pattern of results was unchanged when baseline cognitive composite scores were added as a covariate (Table 4).

A similar pattern of results was also found following a sensitivity analysis in which subjects with a diagnosis of 'Impaired not MCI' were excluded from the group of subjects with normal cognition, with the exception that the association between the dichotomized baseline HAM-D variable and risk of progression also reached significance $(\mathrm{p}=0.043)$ (see supplementary section 4 for additional details of these sensitivity analyses).

Lastly, there was a significant gender $x$ HAM-D interaction for the dichotomous HAM-D measure $(\mathrm{HR}=3.09 ; 95 \% \mathrm{CI}=(1.11,8.65) ; \mathrm{p}=0.031$; for continuous HAM-D: $\mathrm{HR}=1.19$, $95 \% \mathrm{CI}=(0.98,1.45), \mathrm{p}=0.078)$. However, this interaction term did not remain significant when subjects with a diagnosis of 'Impaired Not MCI' were excluded from the group of subjects with normal cognition at baseline $(\mathrm{p}=0.20)$. Cox regression models run separately for males and females (adjusted for baseline age and education) showed no significant association between HAM-D scores and time to symptom onset for either females (for continuous HAM-D: $\mathrm{HR}=1.08,95 \% \mathrm{CI}=(0.98,1.19), \mathrm{p}=0.136$; for dichotomous HAM$\mathrm{D}: \mathrm{HR}=1.97,95 \% \mathrm{CI}=(0.96,4.03), \mathrm{p}=0.063)$ or males (for continuous HAM-D: HR = $0.90,95 \% \mathrm{CI}=(0.77,1.07)), \mathrm{p}=0.239$; for dichotomous HAM-D: $\mathrm{HR}=0.65,95 \% \mathrm{CI}=$ $(0.31,1.36), \mathrm{p}=0.248)$.

\section{DISCUSSION}

We examined the association between depressive symptoms and time to onset of clinical symptoms of MCI in a cohort of individuals with normal cognition at baseline. The primary finding was that within 7 years of baseline, there was an association between depressive symptoms (as measured with the HAM-D) among cognitively normal individuals and time to onset of clinical symptoms of MCI, but not after 7 years from baseline. This association was independent of baseline cognition, gender, education, and age. Of note, this association was found even though the level of depressive symptoms was low (mean $=2.8$ ). Taken 
together, these findings suggest that the presence of depressive symptoms increases the risk of proximal development of MCI due to AD.

Additionally, we sought to understand whether these associations differed by gender, given the increased risk of lifetime depression in women (Kessler et al., 1993). Although we observed an interaction between female gender and dichotomous HAM-D measures on our initial analyses, we did not find a significant association between depressive symptoms and time to onset of clinical symptoms of MCI when analyzed separately for males and females. Furthermore, sensitivity analyses (that excluded subjects with a diagnosis of Impaired not MCI) did not support the presence of the interaction, suggesting a similar association between low-level depression and risk of progression in males and females.

Our finding that low-level depressive symptoms are associated with an increased risk of proximal clinical symptom onset of MCI is consistent with several prior reports. A study of 1821 participants with MCI found that a baseline neuropsychiatric inventory (NPI-Q) (40) score $>0$ increased the risk of incident dementia (Rosenberg et al., 2013). A prospective study of subjects across 30 Alzheimer's Disease Centers in the United States between 2005 and 2011 found that late-life depression but not earlier depression (>2 years before baseline measures) was associated with increased risk of MCI (Steenland et al., 2012). Interestingly, in that study the effect of recent depression did not differ when controlled for antidepressant treatment. Another study found that late-life depression (age $\geq 50$ years) but not earlier depression (age $<50$ years) was associated with increased risk of dementia in cognitively normal subjects (Li et al., 2011). The Rush Religious Orders Study also found that subjects who developed MCI after an average of 3.2 years had significantly higher baseline depressive symptoms (Wilson et al., 2008), though they did not find a significant change in depressive symptom severity prior to MCI symptom onset. While our study did not investigate change in depressive symptom severity, our findings are similar in that higher symptom severity at baseline was associated with increased risk of incident MCI in a similar time-frame.

The effect size of our findings is comparable to prior studies that examined the association of depressive symptoms with incident MCI, though direct comparisons are limited by heterogeneity of study design. Our study is most comparable to the Rush Religious Orders Study, which found an association between incidence MCI and the severity of depressive symptoms measured by the Center for Epidemiologic Studies Depression Scale (CES-D) as a continuous variable $(\mathrm{RR}=1.06 ; 95 \% \mathrm{CI}=(1.002,1.120)$ ) (Wilson et al., 2007).

Studies that examined a diagnosis of depression (as opposed to depressive symptoms) as a risk factor for MCI have reported generally larger effect sizes. For example, Steenland et al., 2012 utilized the Geriatric Depression Scale (GDS) $\geq 5$ and NPI-Q to define depression, and found an association with progression to MCI for subjects who were depressed at first visit, but later not depressed $(\mathrm{RR}=1.41,95 \% \mathrm{CI}=(1.01,1.95), \mathrm{p}=0.04)$, intermittently depressed over follow-up $(\mathrm{RR}=2.22,95 \% \mathrm{CI}=(1.86,2.65), \mathrm{p}<0.0001)$ and always depressed during follow-up $(\mathrm{RR}=2.35,95 \% \mathrm{CI}=(1.86,2.96), \mathrm{p}<0.0001)$. Ravaglia et al., 2008 found an association between depression with antidepressant use and incident MCI $(\mathrm{OR}=2.9,95 \% \mathrm{CI}=(1.3,6.6))$, though the association between depression $(\mathrm{GDS} \geq 10)$ and 
incident $\mathrm{MCI}$ when antidepressant use was not considered did not reach significance $(\mathrm{OR}=$ $1.1,95 \% \mathrm{CI}=(0.7,1.9))$.

The association between late-life depression and development of MCI or dementia has been hypothesized to be secondary to a prodrome (early symptom) of dementia as opposed to a risk factor (predictor) (Panza et al., 2010). Our results are consistent with either explanation as depressive symptoms predicted onset within 7 years after controlling for baseline cognition. It is possible that the onset of mild depressive symptoms in late-life, at a level observed in the current study, reflects a common neuropathological etiology as dementia, leading to proximal cognitive decline, whereas clinically-significant depression that occurs in late life (or earlier) has a different etiology. Further investigation into the relationship of depressive symptomatology and biomarkers of neurodegeneration may provide more insight into these issues.

The current literature on the effect of gender on the association between depression and cognitive decline is yet to be clearly established. Prior longitudinal studies of postmenopausal women have found that subjects meeting criteria for depressive disorder at baseline had an increased risk of incident MCI and dementia (Goveas et al. 2011; Spira et al., 2012). Furthermore, higher depressive symptomatology was associated with increased risk for developing dementia in women but not men in a 16-year cohort study of individuals without cognitive deficits at baseline (Simons et al., 2006). In contrast, a prior study of 1357 community dwelling elderly in the Baltimore Longitudinal Study of Aging found that premorbid depressive symptoms significantly increased risk for dementia, particularly AD, in men but not women (Dal Forno et al., 2005). A similar pattern was found in the PAQUID study, where depressive symptoms at baseline were associated with an increased risk of dementia only in men (Fuhrer et al., 2003). Though many studies investigating the relationship between depression and dementia have controlled for gender, few have separately evaluated gender differences; further studies are needed to address this question.

The study has several important strengths, including: 1) a very long duration of follow up with a mean of 12.5 years and maximum of 20 years, which allows for examining the differential effects of depressive symptoms over a long time-span; and 2) the inclusion of a relatively large number of well-characterized cognitively normal individuals who were primarily in middle-age at baseline. However, the study should also be interpreted within the context of its limitations. For example, participants 1) are well-educated and primarily Caucasian; and 2) had a very low severity of depressive symptoms. Although typical of population samples, this limits generalizability to more severe depressive conditions. As noted above, depressive symptoms of the subjects included in this analysis were far below 8 , the commonly used HAM-D threshold for diagnosis of major depression (Kriston and von Wolff, 2011). Furthermore, data regarding prior diagnosis of depression was not available.

In summary, our results suggest that low levels of depressive symptoms may be predictive of clinical symptom onset of MCI within approximately 7 years among cognitively normal individuals. However, the impact of depressive symptoms on the increased likelihood of progressing to MCI does not appear to extend for longer time intervals. These results suggest that depressive symptoms in middle-aged and older adults may be a useful addition 
to other clinical characteristics such as cognition and genetics (Livingston et al., 2017) in identifying persons at-risk for $\mathrm{MCI}$ due to $\mathrm{AD}$ as part of a thorough clinical evaluation.

\section{Supplementary Material}

Refer to Web version on PubMed Central for supplementary material.

\section{Acknowledgments}

This work was supported by the National Institutes of Health (grant numbers U19-AG033655, P50-AG005146).

The BIOCARD Study consists of 7 Cores with the following members: (1) the Administrative Core (Marilyn Albert, Rostislav Brichko); (2) the Clinical Core (Marilyn Albert, Anja Soldan, Corinne Pettigrew, Rebecca Gottesman, Ned Sacktor, Scott Turner, Leonie Farrington, Maura Grega, Gay Rudow, Daniel D’Agostino, Scott Rudow); (3) the Imaging Core (Michael Miller, Susumu Mori, Tilak Ratnanather, Timothy Brown, Hayan Chi, Anthony Kolasny, Kenichi Oishi, Laurent Younes); (4) the Biospecimen Core (Abhay Moghekar, Richard O'Brien); (5) the Informatics Core (Roberta Scherer, David Shade, Ann Ervin, Jennifer Jones, Hamadou Coulibaly, April Patterson); (6) the Biostatistics Core (Mei-Cheng Wang, Daisy Zhu, Jiangxia Wang); and (7) the Neuropathology Core (Juan Troncoso, Barbara Crain, Olga Pletnikova, Gay Rudow, Karen Fisher). The authors are grateful to the members of the BIOCARD Scientific Advisory Board who provide continued oversight and guidance regarding the conduct of the study including: Drs. John Cernansky, David Holtzman, David Knopman, Walter Kukull, and Kevin Grimm, and Drs John Hsiao and Laurie Ryan, who provide oversight on behalf of the National Institute on Aging. The authors thank the members of the BIOCARD Resource Allocation Committee who provide ongoing guidance regarding the use of the biospecimens collected as part of the study, including: Drs. Constantine Lyketsos, Carlos Pardo, Gerard Schellenberg, Leslie Shaw, Madhav Thambisetty, and John Trojanowski.

The authors acknowledge the contributions of the Geriatric Psychiatry Branch of the intramural program of NIMH who initiated the study (Principle investigator: Dr. Trey Sunderland). The authors are particularly indebted to Dr. Karen Putnam, who has provided ongoing documentation of the Geriatric Psychiatry Branch study procedures and the data files received from NIMH.

\section{References}

Albert M, et al. 2014; Cognitive changes preceding clinical symptom onset of mild cognitive impairment and relationship to ApoE genotype. Current Alzheimer Research. 11:773-784. [PubMed: 25212916]

Albert M, et al. 2018; Progression from normal cognition to mild cognitive impairment for individuals at 5 years. Brain. 141:877-887. [PubMed: 29365053]

Albert M, et al. 2011; The diagnosis of mild cognitive impairment due to Alzheimer's disease: Recommendations from the National Institute on Aging-Alzheimer's Association workgroups on diagnostic guidelines for Alzheimer's disease. Alzheimer's \& dementia. 7:270-279.

Alzheimer's Association. 2017; 2017 Alzheimer's disease facts and figures. Alzheimer's \& Dementia. 13:325-373.

Barnes DE, et al. 2006; Depressive symptoms, vascular disease, and mild cognitive impairment: findings from the Cardiovascular Health Study. Archives of General Psychiatry. 63:273-279. [PubMed: 16520432]

Bhalla RK, et al. 2006; Persistence of neuropsychologic deficits in the remitted state of late-life depression. The American Journal of Geriatric Psychiatry. 14:419-427. [PubMed: 16670246]

Burt DB, Zembar MJ, Niederehe G. 1995; Depression and memory impairment: a meta-analysis of the association, its pattern, and specificity. Psychological bulletin. 117:285. [PubMed: 7724692]

Cummings JL, et al. 1994; The Neuropsychiatric Inventory comprehensive assessment of psychopathology in dementia. Neurology. 44:2308. [PubMed: 7991117]

Dal Forno G, et al. 2005; Depressive symptoms, sex, and risk for Alzheimer's disease. Annals of Neurology. 57:381-387. [PubMed: 15732103]

Dotson VM, Beydoun MA, Zonderman AB. 2010; Recurrent depressive symptoms and the incidence of dementia and mild cognitive impairment. Neurology. 75:27-34. [PubMed: 20603482] 
Feldman H, et al. 2004; Behavioral symptoms in mild cognitive impairment. Neurology. 62:1199-201. [PubMed: 15079026]

Fisher LD, Lin DY. 1999; Time-dependent covariates in the Cox proportional-hazards regression model. Annual review of public health. 20:145-57.

Frank E, et al. 1991; Conceptualization and rationale for consensus definitions of terms in major depressive disorder: remission, recovery, relapse, and recurrence. Archives of General Psychiatry. 48:851-855. [PubMed: 1929776]

Fuhrer R, Dufouil C, Dartigues JF. 2003; Exploring sex differences in the relationship between depressive symptoms and dementia incidence: prospective results from the PAQUID Study. Journal of the American Geriatrics Society. 51:1055-1063. [PubMed: 12890066]

Gabryelewicz T, et al. 2007; The rate of conversion of mild cognitive impairment to dementia: predictive role of depression. International Journal of Geriatric Psychiatry. 22:563-567. [PubMed: 17136705]

Geda YE, et al. 2008; Prevalence of neuropsychiatric symptoms in mild cognitive impairment and normal cognitive aging: population-based study. Archives of General Psychiatry 2008. 65(10): 1193-1198.

Goveas JS, et al. 2011; Depressive Symptoms and Incidence of Mild Cognitive Impairment and Probable Dementia in Elderly Women: The Women's Health Initiative Memory Study. Journal of the American Geriatrics Society. 59:57-66. [PubMed: 21226676]

Hamilton M. 1960; A rating scale for depression. Journal of neurology, neurosurgery, and psychiatry. 23:56.

Hwang TJ, et al. 2004; Mild cognitive impairment is associated with characteristic neuropsychiatric symptoms. Alzheimer Disease \& Associated Disorders. 18:17-21. [PubMed: 15195459]

Kessler RC, et al. 1993; Sex and depression in the National Comorbidity Survey I: Lifetime prevalence, chronicity and recurrence. Journal of Affective Disorders. 29:85-96. [PubMed: 8300981]

Kriston L, von Wolff A. 2011; Not as golden as standards should be: Interpretation of the Hamilton Rating Scale for Depression. Journal of Affective Disorders. 128:175-177. [PubMed: 20696481]

Li G, et al. 2011; Temporal relationship between depression and dementia: findings from a large community-based 15-year follow-up study. Archives of General Psychiatry. 68:970-977. [PubMed: 21893662]

Livingston G, et al. 2017; Dementia prevention, intervention, and care. The Lancet. 390:2673-2734.

Lyketsos CG, et al. 2002; Prevalence of neuropsychiatric symptoms in dementia and mild cognitive impairment: Results from the cardiovascular health study. JAMA. 288:1475-1483. [PubMed: 12243634]

McKhann GM, et al. 2011; The diagnosis of dementia due to Alzheimer's disease: Recommendations from the National Institute on Aging-Alzheimer's Association workgroups on diagnostic guidelines for Alzheimer's disease. Alzheimer's \& Dementia. 7:263-269.

Morris JC. 1993; The Clinical Dementia Rating (CDR): current version and scoring rules. Neurology. 43:2412-2414.

Palmer K, et al. 2010; Neuropsychiatric predictors of progression from amnestic-mild cognitive impairment to Alzheimer's disease: the role of depression and apathy. Journal of Alzheimer's Disease. 20:175-183.

Panza F, et al. 2008; Depressive symptoms, vascular risk factors and mild cognitive impairment. Dementia and geriatric cognitive disorders. 25:336-346. [PubMed: 18319599]

Panza F, et al. 2010; Late-life depression, mild cognitive impairment, and dementia: possible continuum? The American Journal of Geriatric Psychiatry. 18:98-116. [PubMed: 20104067]

Petersen RC, et al. 1999; Mild cognitive impairment: clinical characterization and outcome. Archives of Neurology. 56:303-308. [PubMed: 10190820]

Pettigrew C, et al. 2016; Cortical thickness in relation to clinical symptom onset in preclinical AD. NeuroImage: Clinical. 12:116-122. [PubMed: 27408796]

Ravaglia G, et al. 2008; Prevalent depressive symptoms as a risk factor for conversion to mild cognitive impairment in an elderly Italian cohort. The American Journal of Geriatric Psychiatry. 16:834-843. [PubMed: 18827230] 
Rosenberg PB, et al. 2013; The association of neuropsychiatric symptoms in MCI with incident dementia and Alzheimer disease. The American Journal of Geriatric Psychiatry. 21:685-695. [PubMed: 23567400]

Saczynski JS, et al. 2010; Depressive symptoms and risk of dementia The Framingham Heart Study. Neurology. 75:35-41. [PubMed: 20603483]

Simons LA, et al. 2006; Lifestyle factors and risk of dementia: Dubbo Study of the elderly. Medical Journal of Australia. 184:68. [PubMed: 16411871]

Sperling RA, et al. 2011; Toward defining the preclinical stages of Alzheimer's disease: Recommendations from the National Institute on Aging-Alzheimer's Association workgroups on diagnostic guidelines for Alzheimer's disease. Alzheimer's \& dementia. 7:280-292.

Spira AP, et al. 2012; Depressive symptoms in oldest-old women: risk of mild cognitive impairment and dementia. The American Journal of Geriatric Psychiatry. 20:1006-1015. [PubMed: 22015706]

Steenland K, et al. 2012; Late-Life Depression as a Risk Factor for Mild Cognitive Impairment or Alzheimer's Disease in 30 US Alzheimer's Disease Centers. Journal of Alzheimer's disease: Journal of Alzheimer's Disease. 31:265-275.

Teng E, Lu PH, Cummings JL. 2007; Neuropsychiatric Symptoms Are Associated with Progression from Mild Cognitive Impairment to Alzheimer's Disease. Dementia and geriatric cognitive disorders. 24:253-259. [PubMed: 17700021]

Wilson RS, et al. 2008; Change in depressive symptoms during the prodromal phase of Alzheimer disease. Archives of General Psychiatry. 65:439-445. [PubMed: 18391132]

Wilson RS, et al. 2007; Chronic distress and incidence of mild cognitive impairment. Neurology. 68:2085-2092. [PubMed: 17562829] 


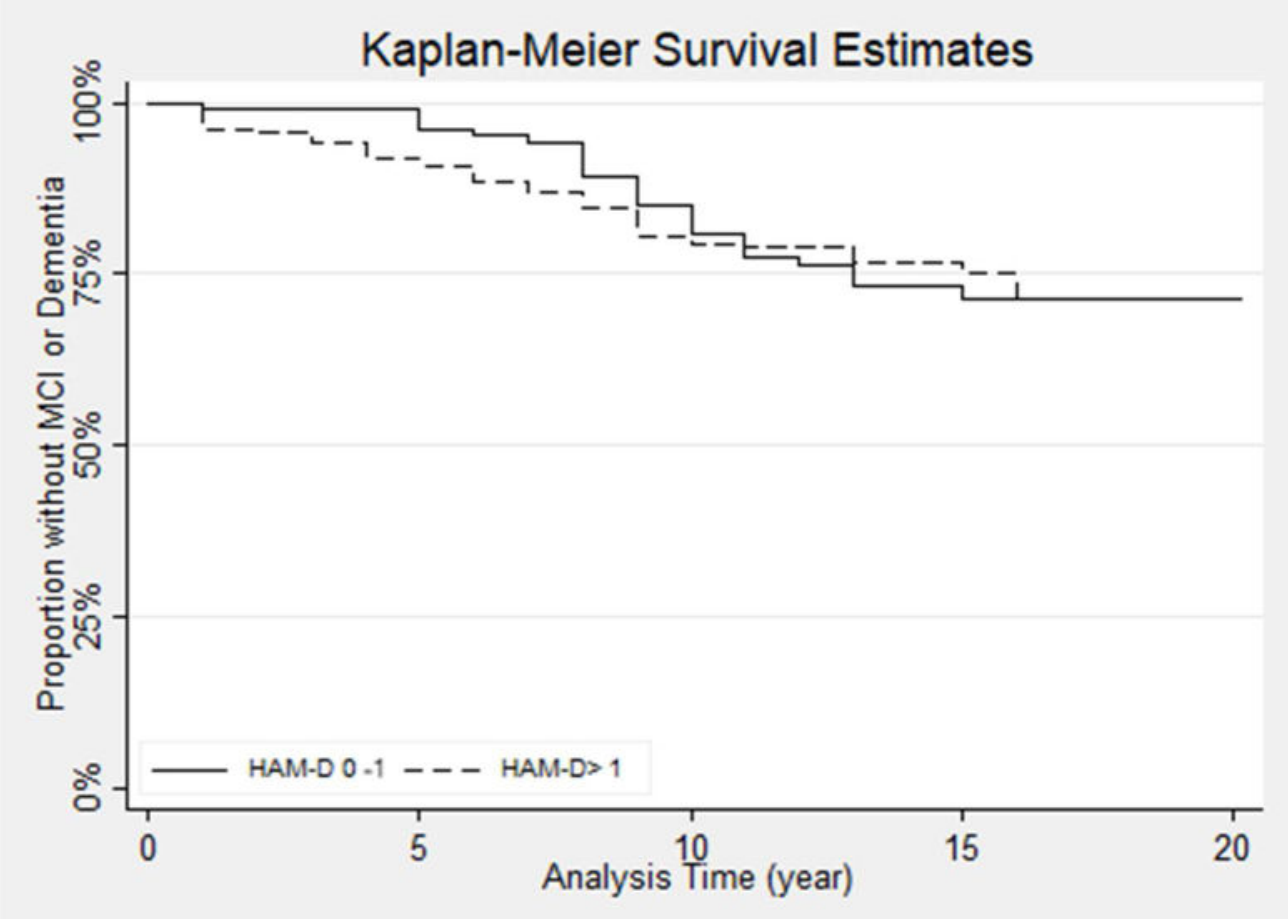

Figure 1.

Kaplan-Meier survival curve plot showing development of incident MCI in those with Hamilton Depression Scale (HAM-D) >1 vs 0-1 


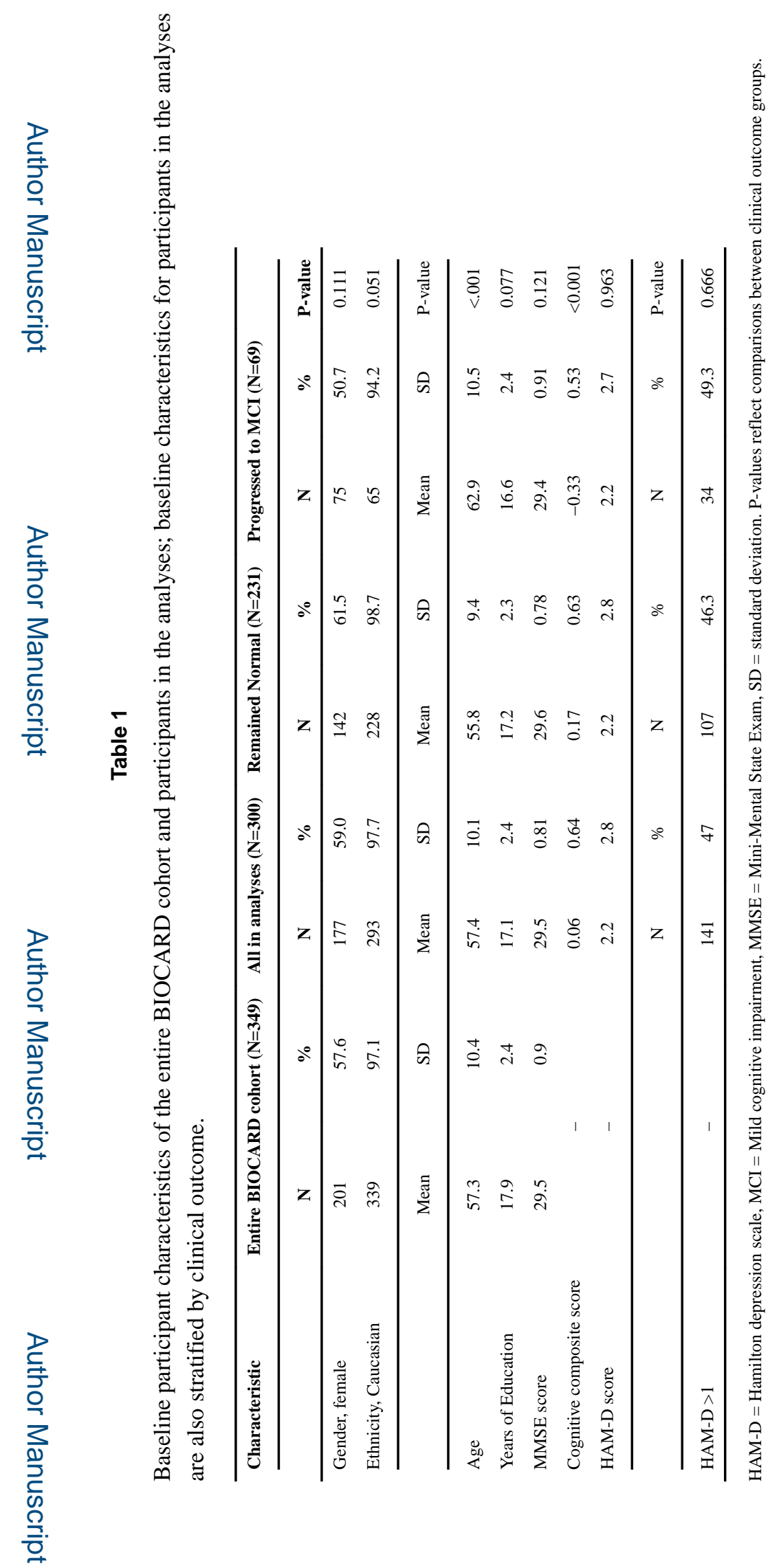

Int Psychogeriatr. Author manuscript; available in PMC 2019 October 01. 


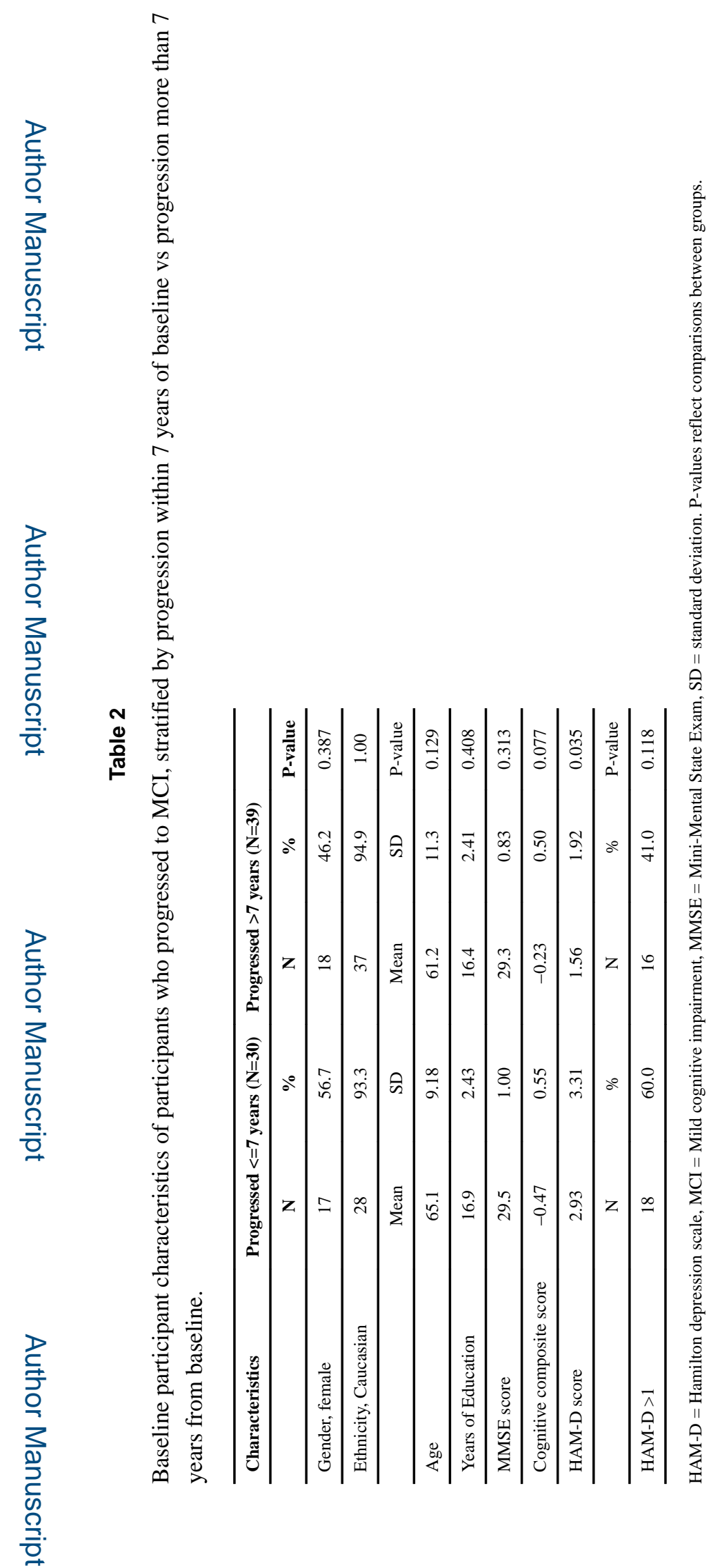

Int Psychogeriatr. Author manuscript; available in PMC 2019 October 01. 


\section{Table 3}

Hazard ratios, 95\% confidence intervals and p-values for baseline Hamilton depression scale scores in relation to clinical symptom onset of MCI, stratified by progression within vs after 7 years from baseline.

\begin{tabular}{lccc}
\hline Variables & HR & $\mathbf{9 5 \%}$ CI & P-value \\
\hline HAM-D continuous & & & \\
\hline Progression < 7 years & 1.11 & $1.00,1.23$ & 0.043 \\
\hline Progression > 7 years & 0.91 & $0.79,1.05$ & 0.194 \\
\hline HAM-D > 1 vs 0-1 & & & \\
\hline Progression < 7 years & 1.83 & $0.88,3.81$ & 0.108 \\
\hline Progression > 7 years & 0.79 & $0.42,1.50$ & 0.471 \\
\hline
\end{tabular}

Separate models were run for the continuous and dichotomous HAM-D scores. Models were adjusted for gender, baseline age, and education. HAM-D = Hamilton Depression Scale, CI = Confidence Interval, $\mathrm{HR}=$ hazard ratio, $\mathrm{MCI}=$ mild cognitive impairment. 


\section{Table 4}

Hazard ratios, 95\% confidence intervals and p-values for baseline Hamilton depression scale scores and baseline cognitive composite scores in relation to clinical symptom onset of MCI within vs after 7 years from baseline.

\begin{tabular}{lccc}
\hline Variables & HR & $\mathbf{9 5 \%}$ CI & P-value \\
\hline HAM-D continuous & & & \\
\hline Progression < 7 years & 1.14 & $1.02,1.28$ & 0.028 \\
\hline Progression $>7$ years & 0.91 & $0.77,1.07$ & 0.268 \\
\hline Cognitive composite score & 0.29 & $0.18,0.47$ & $<0.001$ \\
\hline HAM-D $>1$ vs 0-1 & & & \\
\hline Progression $<7$ years & 1.90 & $0.84,4.30$ & 0.122 \\
\hline Progression $>7$ years & 0.79 & $0.40,1.56$ & 0.504 \\
\hline Cognitive composite score & 0.29 & $0.18,0.47$ & $<0.001$ \\
\hline
\end{tabular}

Separate models were run for the continuous and dichotomous HAM-D scores, adjusted for gender, baseline age, and education. HAM-D = Hamilton Depression Scale, CI = Confidence Interval, $\mathrm{HR}=$ hazard ratio, $\mathrm{MCI}=$ mild cognitive impairment. 